\title{
STEM Fluctuation Microscopy Characterization of an Amorphous - Protocrystalline Metal Glass Nanocomposite
}

Feng Yi, Jinwoo Hwang, Seth Imhoff, J. H. Perepezko, and P. M. Voyles

Department of Materials Science and Engineering, University of Wisconsin, Madison, 1509 University Ave, Madison, WI 53706-1595

At a length scale of a few nanometers, the structure of metallic glasses may be heterogeneous, consisting of regions of greater structural order and disorder. Deliberate nanocomposites, in which a crystalline phase is created in the glassy matrix by an in-situ reaction, have been used in metallic glasses for magnetic [1] and structural [2] applications. We have studied the structure of a set of high Al-content metallic glasses, which are naturally-occurring "nanocomposites". In their asquenched state, these materials contain quenched-in protocrystalline nuclei: subcritical clusters with crystalline or near-crystalline atomic order [3]. On heating, these clusters can act to catalyze the primary crystallization reaction, converting a portion of the surrounding glassy material to the stable crystal phase and creating a true nanocomposite.

Fluctuation electron microscopy (FEM) is ideally suited to studying amorphous / nanocrystal composites, which can be hard to study by HRTEM or HRSTEM because the amorphous material creates such a large background that the contrast from very small nanocrystals is lost. In FEM, we use systematic coherent electron nanodiffraction to study nanometer-scale structure in amorphous materials. The fundamental dataset is $I(\mathbf{r}, \mathbf{k}, Q)$, the diffracted intensity as function of position on the sample $\mathbf{r}$, diffraction vector $\mathbf{k}$, and microscope reciprocal space resolution $Q$. We then compute the normalized variance of the diffracted intensity,

$$
V(\mathbf{k}, Q)=A\left(\int I^{2}(\mathbf{r}, \mathbf{k}, Q) d^{2} \mathbf{r} /\left[\int I(\mathbf{r}, \mathbf{k}, Q) d^{2} \mathbf{r}\right]^{2}\right)-1,
$$

where the integral covers the area $A$ of the sample. $V$ is a measure of spatial heterogeneity in diffraction, and thus in structure. The diffracted intensity from a crystal scales as the square of the number of atoms in the crystal, so even small crystals give rise to large excursions in the local diffracted intensity, and thus a large $V$.

Fig. 1(a) shows $V(k)$ measured at $Q=0.34 \mathrm{~nm}^{-1}$, which corresponds to a real-space resolution of 1.8 $\mathrm{nm}$. The peaks in $V(k)$ occur at the positions of the $\mathrm{Al}\{111\},\{200\}$, and $\{220\}$ Bragg reflections. This, combined with diffraction simulations [3], shows that the primary form of nanoscale order in these materials is small regions with the Al crystal structure. Fig. 1(a) also shows that small changes in composition make a large changes in structure. Substitution of 1 at.\% $\mathrm{Cu}$ for $\mathrm{Y}$ increases $V(k)$ at the $\mathrm{Al}\{111\}$ position and creates clearer peaks at the $\{200\}$ and $\{220\}$ positions. Substitution of 1 at.\% $\mathrm{Cu}$ for $\mathrm{Al}$ suppresses $V(k)$. Fig 1(b) shows differential scanning calorimetry measurements of the crystallization of similar samples. The onset of the primary crystallization reaction shifts to lower temperature for samples with high $V(k)$ and a more heterogeneous structure, and to higher temperature for the sample with lower $V(k)$. The changes in $V(k)$ are caused by changes in the size and density of the quenched-in nuclei that drive the phase transformation. We have recently developed a heterogeneous nucleation theory model that connects this structure to the changes in the crystallization reaction [4]. 

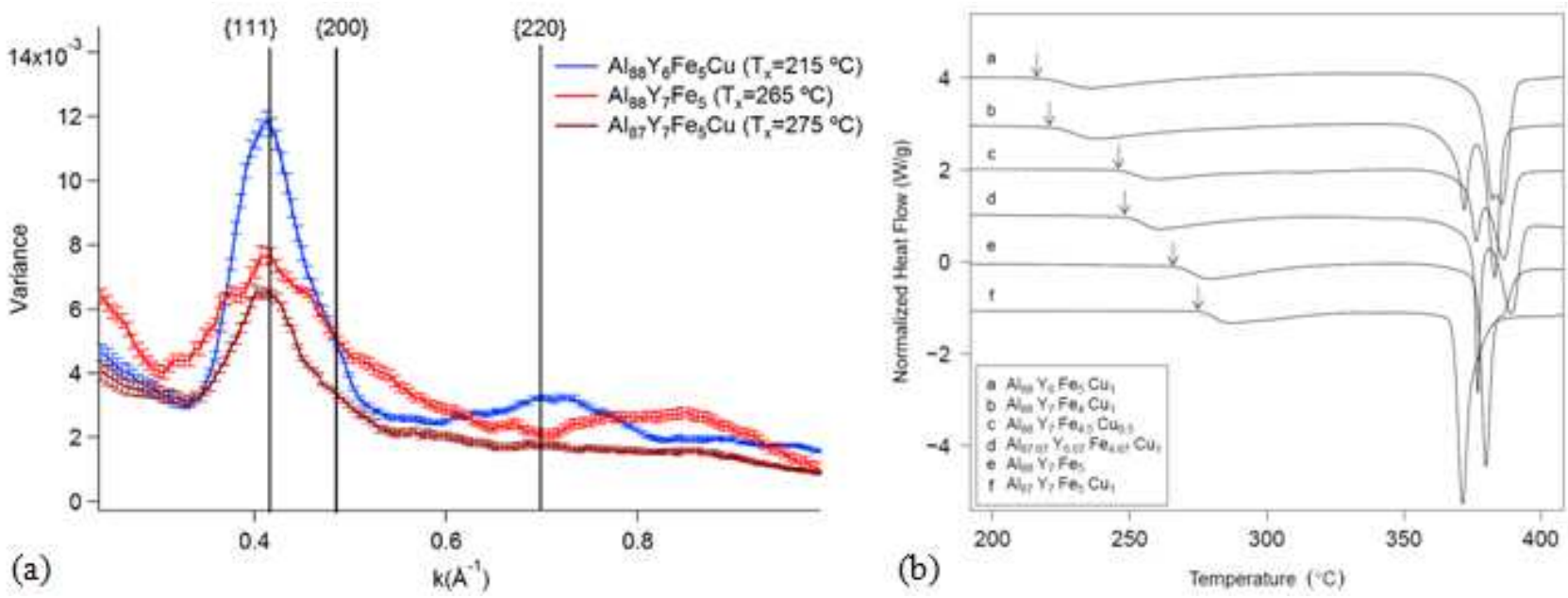

Fig. 1(a) FEM data acquired from three high Al-content metallic glasses in the as-quenched state. Substitution of 1 at.\% $\mathrm{Cu}$ make large changes in the structure of the material. (b) Differential scanning calorimetry of the primary crystallization reaction of similar samples. The arrows mark the primary crystallization onset temperatures, which also show large changes with $\mathrm{Cu}$ substitution.

Unlike our previous experiments, which were carried out using dark-field TEM imaging [3], the data in Fig. 1 were collected using STEM nanodiffraction. STEM FEM has reduced cross-talk between positions on the sample and substantially better coherence, both of which increase $V(k)$. The coherence of the nanodiffraction probes on our probe aberration-corrected Titan (S)TEM is shown in Fig. 2. Fig. 2(b) shows the source size at the sample determined by fitting the real-space image of the probe to a convolution of an Airy function probe and an incoherent Gaussian source [4]. Fig. 2(c) shows that the probe current is linear in the source area, reinforcing that the best coherence is achieved only at the lowest current.

\section{References}

[1] M. A. Willard, V. G. Harris, JOM 54, 44 (2002).

[2] see e.g. C. C. Hays, C. P. Kim, and W. L. Johnson, Phys. Rev. Lett. 84, 2901 (2000).

[3] W.G. Stratton, J. Hamann, J.H. Perepezko, P.M. Voyles, X. Mao and S.V. Khare, Appl. Phys. Lett. 86, 141910 (2005).

[4] S. Imhoff, F. Yi, P. M. Voyles, and J. H. Perepezko (unpublished).

[4] F. Yi, P. Tiemeijer, and P. M. Voyles, J. Elec. Microsc. (to be published).

[5] This research was supported by the NSF (DMR-0905793 and 1005334 and CMMI-0824719).
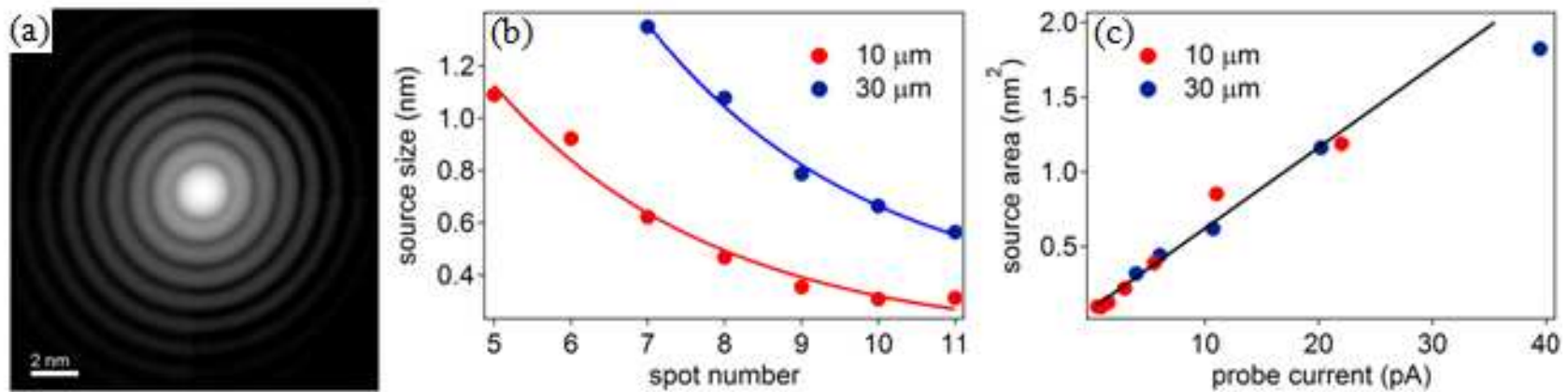

Fig. 2(a) An image of a highly coherent, $1.2 \mathrm{mrad}$ convergence probe; (b) the incoherent source size at the sample for a set of $1.2 \mathrm{mrad}$ probes at different spot number and aperture size; (c) linear scaling of the source area and current. 